\title{
Synaptic Arrays of the Inner Plexiform Layer in the Developing Retina of Xenopus
}

\author{
LESLIE J. Fisher \\ Department of Anatomy, University of Michigan Medical School, Ann Arbor, Michigan 48109
}

Accepted January 20, 1976

\begin{abstract}
During embryonic and larval development in Xenopus laevis, arrays of synapses made by amacrine cells form in two phases: an initial phase of rapid synaptic addition and a second phase of slower addition. In the region near the optic nerve, at which all measurements were made, these synapses first appear at stage 40 (approx $66 \mathrm{hr}$ postfertilization). Connectivity increases at a rate of 8.6 synapses per hr per inner nuclear layer (INL) nucleus until stage 47 (132 $\mathrm{hr}$ postfertilization). After this phase the rate of formation decreases to 1.19 synapses per hr per INL nucleus. Synaptic arrays made by bipolar cells have only one phase of addition. A synapse made by a bipolar cell may be identified by its presynaptic ribbon, the first of which are seen at stage 40. Ribbons are added to the IPL neuropil at a rate of 4.6 ribbons per hr per INL nucleus until stage 47. After this the number of ribbons per INL nucleus in the area near the optic nerve remains constant. Although they may be found, amacrine to amacrine synapses (serial conventional) remain at low numbers throughout larval and early postmetamorphic life. This is unlike the condition found in Rana pipiens where a dramatic increase in amacrine to amacrine connectivity occurs at metamorphosis.
\end{abstract}

\section{INTRODUCTION}

In 1968 Dowling published a quantitative study of the synaptic organization of the retina of adult primates and frogs. His data demonstrated that through careful measurement quantitative differences could be discovered between qualitatively similar retinas. Since that time, other investigators have utilized quantitative descriptions of inner plexiform layer (IPL) synapse densities in other amphibians, rabbits, rats, and pigeons (Dubin, 1970; Sosula and Glow, 1970; Yazulla, 1974). All of these investigators utilized adult tissue.

Developmentally oriented studies of retinal organization for the most part have been qualitative. Studies of the relative time of first appearance of bipolar and amacrine synapses or of receptor synapses have been made in Rana (Nilsson and Crescitelli, 1969, 1970), rat (Weidman and Kuwabara, 1968), and mouse (Olney, 1968). Quantitative methods of analysis were first applied to the development of retinal synapses by Fisher (1972). No quantitative description of the appearance of IPL synapses has yet included the entire developmental period from the time of their first appearance to that of the fully matured retina.

Several questions of interest present themselves in such a quantitative developmental study: What are the temporal sequences in which the mature IPL synaptic arrays were formed? What is the rate of formation of new synapses? How does the physical growth of retinal volume affect the times and rate of synaptic appearance? A detailed quantitative description of the normal sequence of synaptic formation during retinogenesis provides a groundwork for investigating factors which influence the normal development of synaptic arrays. What environmental or intrinsic factors affect the normal rates and sequences of synapse development? Answers to these questions concerning the retina 
may well provide insight into determinants of synaptogenesis elsewhere in the central nervous system.

In the present study, both electron and light microscopy were utilized to obtain a normal profile of the development of retinal synaptic arrays in the anuran amphibian Xenopus laevis. Density of IPL synapses per unit volume made by bipolar and amacrine cells was computed for several stages of maturation. In addition, measurements were made of various physical parameters involved with IPL and inner nuclear layer (INL) growth and differentiation. These data were used to determine both the numerical density of synapses and the number of synapses per neuron as a function of developmental stage. The resulting growth curves are an attempt to describe the dynamics of synapse array formation during retinogenesis.

\section{MATERIALS AND METHODS}

In the course of study, larval Xenopus of stages 30 through 66 (Nieuwkoop-Faber, 1967) were obtained from the University of Michigan Amphibian Center where eggs, obtained by induced ovulation, were fertilized, hatched, and grown under a standard set of conditions (Nace et al., 1974). Each tadpole was carefully staged according to the criteria of Nieuwkoop and Faber (1967). Animals were anesthetized in a $0.05 \%$ aqueous solution of tricaine methane sulphonate. The eyes were removed and fixed for $1.33 \mathrm{hr}$ in $0.067 \mathrm{M}$ veronal acetate-buffered $1.3 \%$ osmium tetroxide at pH 7.8. Fixation was followed by overnight staining en bloc with $0.5 \%$ aqueous uranyl acetate. After dehydration in graded alcohols the eyes were embedded in Epon. Thick $(60 \mu \mathrm{m})$ sections were taken (West, 1972) and the section passing through the optic nerve was remounted on a plastic block. Sections (1- and 5- $\mu \mathrm{m}$ thick) were taken for light microscopy and sections 600 - to $900-\AA$ thick were taken and mounted on uncoated bar grids for electron microscopy. The line of the choroidal fis- sure allows the eye to be oriented so that the area of retina to be sampled could be localized to within $200-\mu \mathrm{m}$ medial or lateral to the optic nerve head. This retinal locus was used for all measurements. No distinction was made between retina medial or lateral to the optic nerve. During thin-sectioning, note was taken of the section's interference color. The inferred section thickness was used in the subsequent data processing (Peachey, 1958). Not all animals used were from a single clutch of eggs, nor were the stages timed. Average times from fertilization for each stages were taken from Nieuwkoop and Faber (1967).

\section{Electron Microscopy}

Contiguous electron micrographs, covering the thickness of the inner plexiform layer from the ganglion cell layer to the inner nuclear layer, were prepared for each animal. The micrographs were assembled into a mosaic, at a final magnification of $26,000 \times$, the magnification of which was standardized by including a micrograph of a calibration grid (Fullam \#1002) in each session. Photographic magnification was standardized for each printing session.

A polar planimeter was used to measure the area of inner plexiform layer covered by each mosaic. The scleral edge of the ganglion cell perikarya and the vitreal edge of the inner nuclear layer defined the vitreal and scleral boundaries of the sample area while the lateral boundaries were arbitrary.

Amacrine cell synapses were identified by a combination of membrane specialization and a subjacent accumulation of synaptic vesicles (Fig. 1). These synapses were subdivided into two separate and mutually exclusive sets: conventional and conventional in serial configuration. The difference between these two sets is that the former is presynaptic to a process that does not contain a conventional synapse within that plane of section (unnumbered 


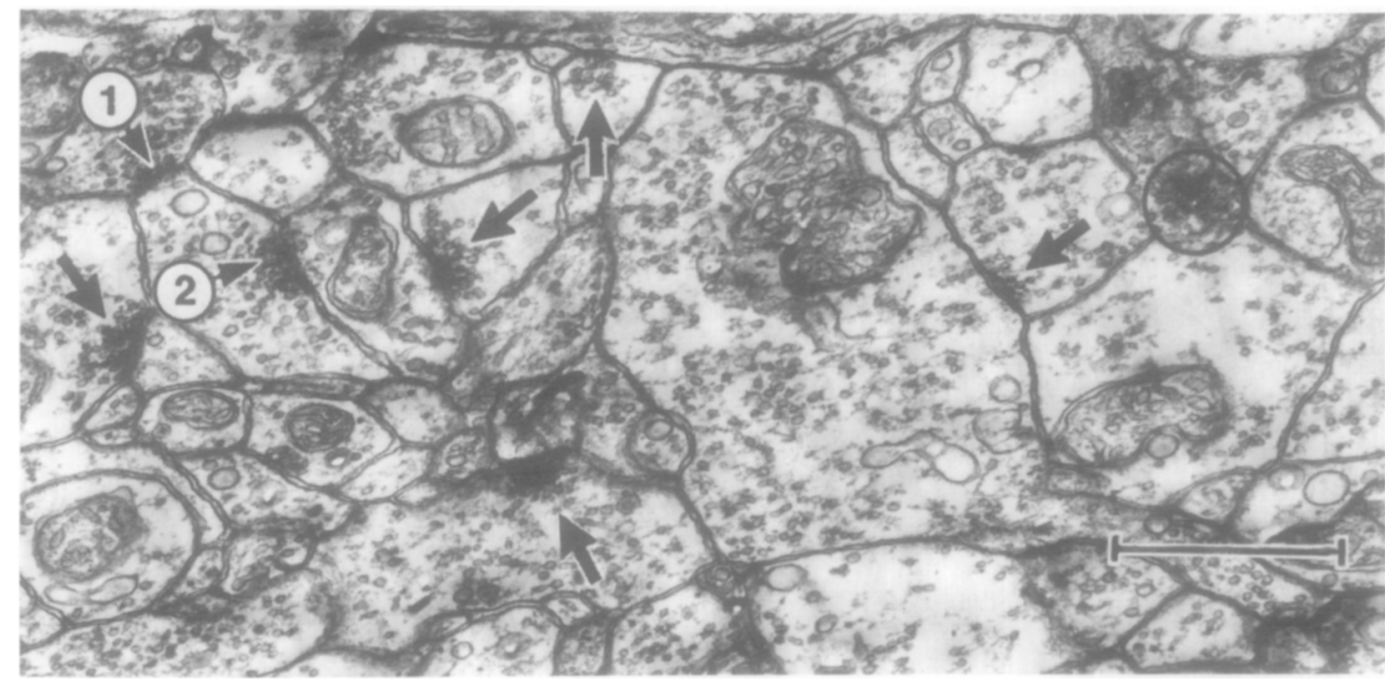

FIG. 1. A survey electron micrograph of inner plexiform layer. This micrograph is a portion of a mosaic that was used in identifying and quantifying synapses. Conventional synapses (arrow), conventional synapses in serial configuration ( 1 and 2 ), and a synaptic ribbon (encircled) are seen. Calibration bar equals $1 \mu \mathrm{m}$.

arrows, Fig. 1), whereas the latter are characterized as being pre- and postsynaptic conventional synapses by and onto a single process (numbered arrows, Fig. 1). Each of the synapses identified as being members of a serially conventional group was counted as an individual synapse. Thus two conventional synapses in serial configuration were recorded as two synapses within the serial subset, not as one pair of conventional synapses.

Bipolar cell synapses were identified by the presence of a presynaptic ribbon surrounded by synaptic vesicles (encircled in Fig. 1; arrow in Fig. 2). Membrane modifications, while usually present, were not regarded as being an essential feature within the plane of section. While there is some disagreement as to the presence of synapses of the conventional type within biopolar terminals, this study assumes ribbons to be the exclusive synaptic form within bipolar processes.

The length of ribbons and postsynaptic membrane specializations were measured using dial calipers with a resoluton equivalent to $19 \AA$ on the mosaic. A modified Abercrombie procedure was used to correct for sampling error due to section thickness

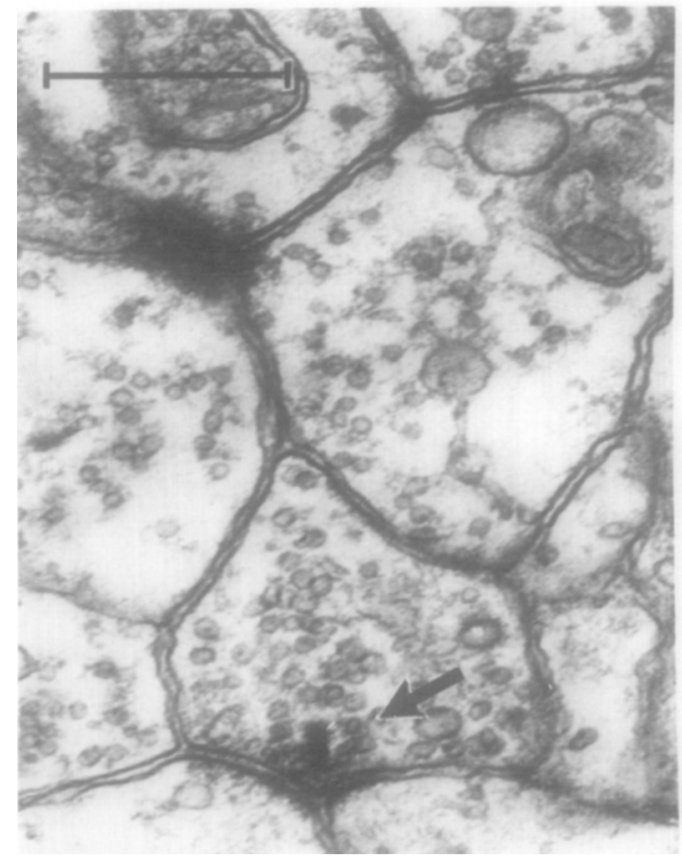

FIG. 2. IPL synaptic ribbon. The characteristic dimension of the ribbon which was used in the modified Abercrombie connection was its length rather than postsynaptic membrane specialization. Calibration bar equals $0.5 \mu \mathrm{m}$.

and synaptic or ribbon size (Dubin, 1970). Densities in numbers of synapses or ribbons per unit volume were computed from 
these data for 14 stages of larval growth. One eye from each of 47 animals were used to obtain synaptic density measurements (Table 1).

\section{Light Microscopy}

One-micrometer-thick sections of Eponembedded retina were stained with methylene blue and examined to insure that the plane of section was normal or nearly normal to the plane of the IPL. Thicknesses of inner plexiform layer and of inner nuclear layer were then measured at a magnification of $400 \times$ using a filar micrometer.

Inner nuclear layer nuclei were counted on adjacent 1 - and 5- $\mu \mathrm{m}$ methylene bluestained sections under an oil immersion objective at $1000 \times$ magnification. These data were then corrected for sampling error due to section thickness by the method of Ebbisson and Tang (1965). The inner nuclear layer areas covered by these counts were measured by polar planimetry of photographs at $400 \times$ magnification. Densities of INL nuclei per unit volume were calculated using these data.

RESULTS

\section{Light Microscopy}

Both IPL and OPL have well-defined boundaries at stage 37 , the youngest stage

TABLE 1

Number of Animals and Total Mosaic Sample Area Used to Obtain Synapse Density

\begin{tabular}{|c|c|c|}
\hline Stage & $\begin{array}{l}\text { Number of an- } \\
\text { imals used per } \\
\text { stage }\end{array}$ & $\begin{array}{l}\text { Total sample } \\
\text { area }\left(\mu \mathrm{m}^{2}\right)\end{array}$ \\
\hline 37 and younger & 5 & Not measured \\
\hline 40 & 3 & 1606 \\
\hline 41 & 5 & 1794 \\
\hline 42 & 3 & 1708 \\
\hline 45 & 5 & 2056 \\
\hline 46 & 4 & 2672 \\
\hline 47 & 2 & 1672 \\
\hline 48 & 3 & 1801 \\
\hline 49 & 3 & 1919 \\
\hline 51 & 1 & 770 \\
\hline 55 & 3 & 1869 \\
\hline 57 & 4 & 3063 \\
\hline 59 & 2 & 1573 \\
\hline Juvenile & 4 & 3039 \\
\hline
\end{tabular}

utilized in this study. Figure 3 is representative of the appearance of the eye at these early stages. All retinal layers are present with neuronal differentiation well advanced. The optic nerve is present indicating that ganglion cells of the central retina have established axons. Receptor outer segments have appeared and are starting to elongate. Each receptor inner segment contains a refractile vacuole or droplet.

Ganglion cells are quite closely packed within the ganglion cell layer (GCL) and are often two nuclei deep. The entire eye

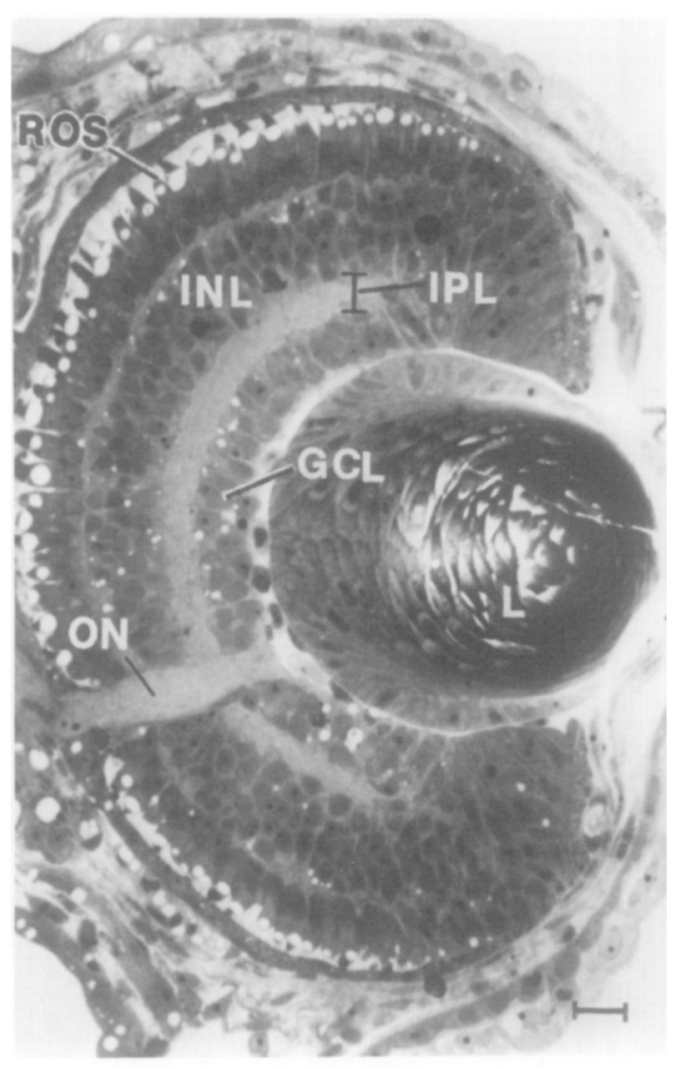

FIG. 3. Cross section of stage 42 Xenopus eye at the optic nerve. Cross section of stage 42 Xenopus eye ganglion cell layer (GCL), inner plexiform layer (IPL), inner nuclear layer (INL), and receptor outer segments (ROS) are all well-defined, although the outer segments are still quite small. The calibration bar equals 10 micrometers. This section passes directly through the optic nerve (ON) as did all sections that were used for study. 
at stage 42 is less than $0.4 \mathrm{~mm}$ across at its largest diameter.

The well-defined boundaries of IPL and INL allow measurements of their respective thickness to be made with accuracy, even at early stages. The thickness of the IPL at stage 42 is approximately $17 \mu \mathrm{m}$ from the edge of the ganglion cell layer to the border of the nuclear layer (Fig. 4). At this same stage the INL measures approx $25 \mu \mathrm{m}$ from its border with the IPL to its border with the outer plexiform layer (Fig. 5). Nuclei of this layer are closely packed with a mean corrected density at stage 42 of 0.55 nuclei per $1000 \mu \mathrm{m}^{3}$ (Fig. 6).

At stage 57 extensive changes from the younger retina can be seen (Fig. 7). Receptor outer segments have lengthened considerably and now only cones contain an elipsoid droplet. Ganglion cells are less densely packed and form an interrupted single layer of nuclei. The eye has grown in its total diameter to approx $1.5 \mathrm{~mm}$.

Total volume of both IPL and INL increase as the larva grows. This increase is the result of two factors: the increase in total retinal area by growth at the retinal margins and an increase in thickness. At stage 57 the thickness of the IPL at the specific retinal locus used for measure-

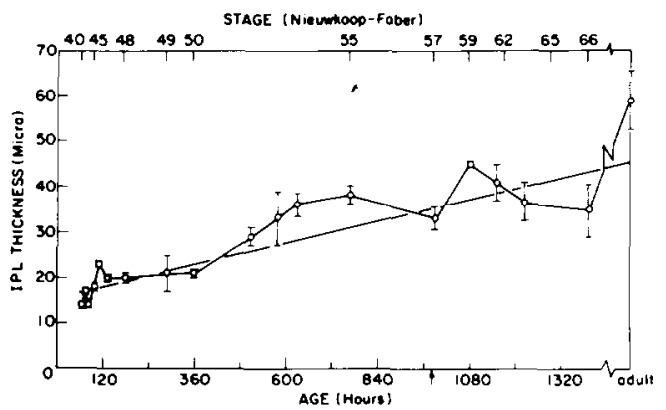

FIG. 4. Inner plexiform layer (IPL) thickness. IPL thickness is plotted as a function of age and of larval stage. The arrow denotes the stage of forelimb emergence. On this and all subsequent figures where they appear, error bars indicate \pm 1 SEM. The straight line plotted through the data points in Fig. 4 and 5 are linear regressions calculated by the method of least square for their respective data. In total, 68 animals were used for these data.

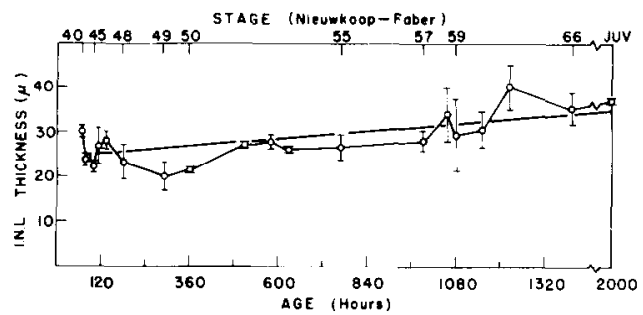

FIG. 5. Inner nuclear layer thickness. INL thickness is plotted as a function of age and larval stage.

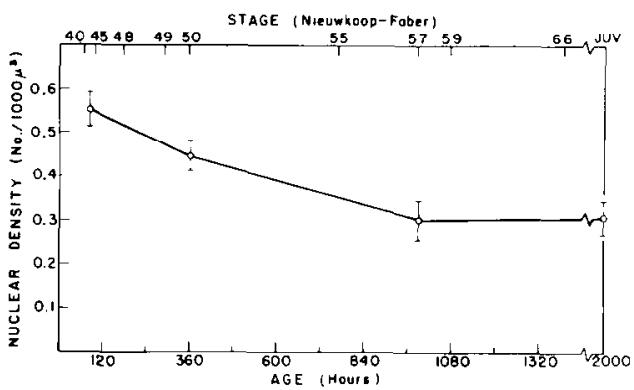

FIG. 6. INL nuclear density. Nuclei of the INL were counted and the number corrected for sampling error before calculation of nuclear density. The resultant nuclear density is plotted as a function of age and larval stage.

ment is about $30 \mu \mathrm{m}$, an increase of $13 \mu \mathrm{m}$ from stage 42 (Fig. 4) while the thickness of the INL is approx $31 \mu \mathrm{m}$, an increase of $6 \mu \mathrm{m}$ (Fig. 5). Density of nuclear packing in the INL, however, decreases over the same period to a mean value of about 0.31 nuclei per $1000 \mu \mathrm{m}^{3}$ (Fig. 6). This decrease may be due to several factors including growth of nuclear size, an increase in internuclear substance, neuronal death or migration, or a combination of these factors.

\section{Electron Microscopy}

Conventional Synapses. Conventional synapse density (numbers of synapses per unit volume) expressed as a function of larval age reveals two distinct phases in the formation of synaptic arrays (Fig. 8). Sixty-six hours postfertilization, corresponding to stage 40 , marks the beginning of the formation of synaptic arrays in the central retina. The density of conventional 


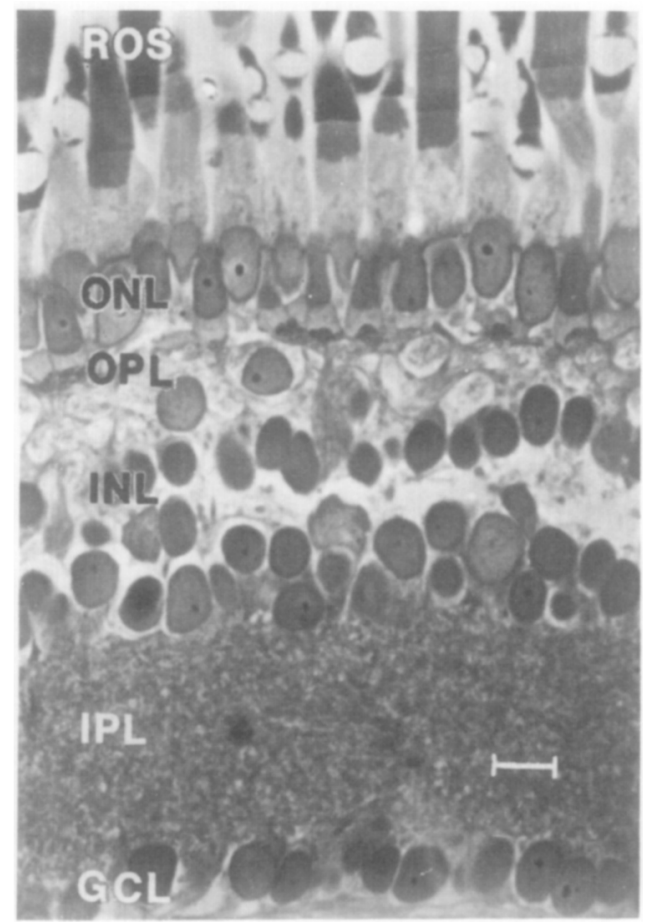

FIG. 7. Cross section of stage 57 retina near optic nerve. All layers are visible. The IPL is wider and the receptor outer segments are longer than the stage 37 eye (Fig. 1). Both rods and cones can be distinguished. The ganglion cell layer has been reduced to one nuclear thickness. Calibration bar equals $10 \mu \mathrm{m}$. Receptor terminals in the outer plexiform layer (OPL) are clearly seen.

synapses increases rapidly until stage 47 , $132 \mathrm{hr}$ postfertilization. In $66 \mathrm{hr}$, the number of conventional synapses made by amacrine cells increases from none to nearly 500 synapses per $1000 \mu \mathrm{m}^{3}$. Following $132 \mathrm{hr}$ conventional synaptic densities change more slowly.

From the data given in Figs. 4-6, and 8, it is possible to calculate values for the absolute number of conventional synapses per INL nucleus and rates of synapse production per nucleus per unit time (see Fig. 9 and its legend for the method of calculations). While these data do not give synapses per amacrine cell, they are proportional to the number per amacrine cell provided that there are no nuclei added to or subtracted from the INL at the sample

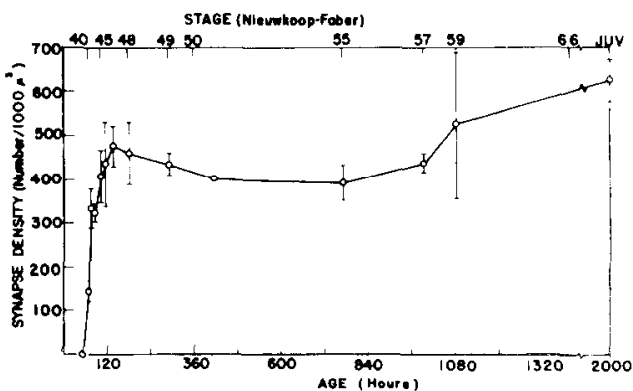

Fig. 8. Conventional synapse density. Densities of conventional synapses are expressed as number of synapses per $1000 \mu \mathrm{m}^{3}$ and plotted as a function of age. Since the IPL increases in volume as a function of age, these data do not directly represent the total number of synapses present in the IPL. They are, rather, the average number of synapses found in a unit volume of neuropil.

locus. Since there are nuclei other than amacrine cell nuclei located in the INL, expressing the data as synapses per INL nucleus gives the lowest possible figure for the number of conventional synapses per amacrine cell.

The highest rate of synapse production, 8.6 conventional synapses per hr per nucleus, occurs between stage 40 and 47 . The overall rate of production following stage 47 is 1.19 conventional synapses per $\mathrm{hr}$ per nucleus. The mean number of synapses per nucleus in the post-stage 47 larva changes from 700 to 2800: a fourfold increase.

Synaptic ribbons. The synaptic ribbons of bipolar cell processes reach a peak density of 270 ribbons per $1000 \mu \mathrm{m}^{3}$ at stage 47, $132 \mathrm{hr}$ postfertilization (Fig. 10). The density of these synaptic elements decreases following stage 47 to the approx 70 ribbons per $1000 \mu \mathrm{m}^{3}$ found in the juvenile.

These same data are presented as the number of ribbons per INL nucleus in Fig. 11. From stage 40 until stage 47 , each biopolar neuron increases its complement of synaptic ribbons. Following stage 47 , however, there is essentially no net change in the number of synaptic ribbons per neuron. Thus ribbons are manufactured at a rate of 4.6 ribbons per hr per INL nucleus 


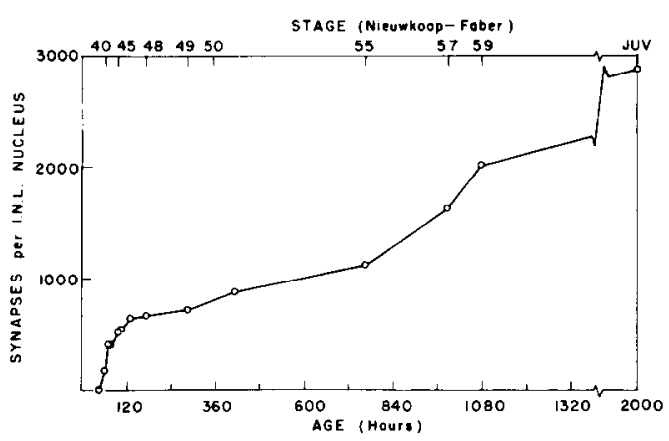

Fig. 9. Conventional synapses per INL nucleus. These are the same data as plotted in Fig. 8, but with the additional information of IPL and INL growth used to compute the number of synapses per INL nucleus at each sampled stage. Explanation of computation: To compute the total number of synapses per neuron, the regression fit of the data given in Fig. 4, IPL thickness, was multiplied by the density of synapses at corresponding ages (Fig. 8) to yield the total number of synapses present in a vitreo-sclerad column of IPL of unit cross-sectional area. The same procedure was used with nuclear density and the INL thickness to calculate the total number of INL nuclei in a column of unit crosssectional area. If the nuclear and synaptic density can be considered to be homogeneous throughout the area covered by several columns at this retinal locus, the synapses calculated for any column will, on the average, be equal to the number of synapses made by the nuclei contained in the same column. This procedure has, in effect, divided the retina into columns with a vitreo-sclerad orientation and a cross section of unit area. The number of IPL synapses in that column represent the average number of synapses made by the nuclei found in that column. This is not to say that each nucleus is associated with the synapses in the directly subjacent IPL column. The ramifications of processes within the IPL covers an area beyond the unit cross-sectional area but, on the average, the synapses found in any column will be numerically equal to the number of synapses made by the nuclei in that column. The number of synapses per column was then divided by the number of nuclei per column to give the total number of synapses per nucleus.

until stage 47 at which time the net rate of ribbon production goes to zero.

Conventional synapses in serial configuration. Conventional synapses in serial configuration have a much lower density and exhibit more variation in occurrence at all stages than either conventional synapses or ribbons (Fig. 12). When expressed

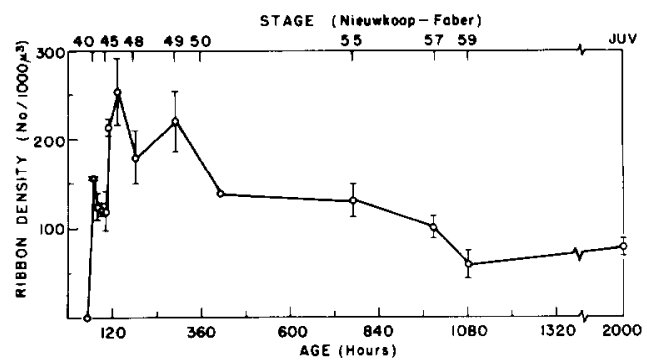

FIG. 10. Ribbon density. The breakpoint at stage 47 is pronounced but the apparent decrease in density thereafter does not reflect a net loss of ribbons with stage (see Fig. 11).

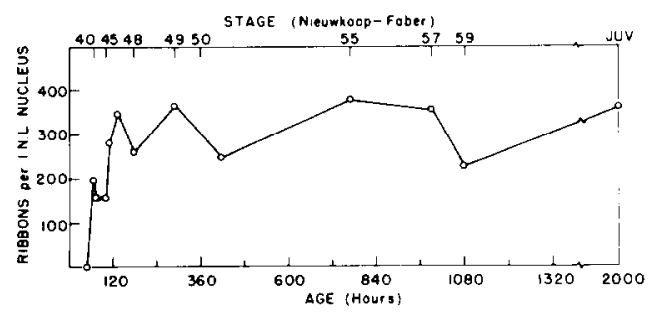

FIG. 11. Ribbons per INL nucleus. Both IPL and INL growth account for the decrease in density seen in Fig. 10. This growth profile demonstrates that the net number of ribbons per INL nucleus remains constant after stage 47 .

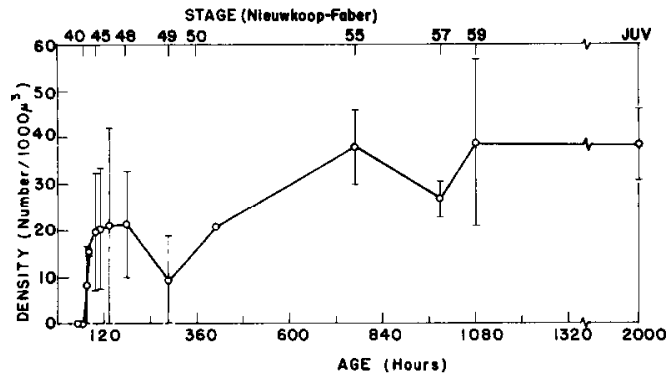

FIG. 12. Serial configuration synapse density. Conventional synapses in serial configuration remain at low density throughout larval and juvenile life. Large variations in densities appear from animal to animal.

as synapses per neuron (Fig. 13) serial synapses increase in a fairly linear fashion at a rate of 0.4 synapses per hr per INL nucleus.

Growth in thickness of both IPL and INL continues throughout larval and early juvenile life. Nuclear density within the INL, however, remains approximately 


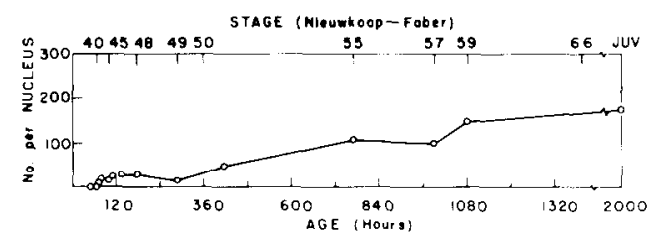

Fig. 13. Serial conventional synapses per INL nucleus. As the larvae grow, amacrine to amacrine connections are slowly added. No dramatic change in their number nccurs at stage 57 (metamorphic climax).

constant after stage 57 (Fig. 6).

Dynamic aspects of growth may be appreciated by consideration of the data when plotted on a linear time scale (lower abscissa, Figs. 4-6) rather than as a function of developmental stage (upper abscissa, Figs. 4-6). Growth profiles of both IPL and INL may be approximated by a linear increase with time. A least squares linear regresion of the IPL growth profile (Fig. 4) is given by the equation

$$
T_{\text {IPL }}=0.02 h+15.8
$$

where $T_{\text {IPI }}$ is the thickness of the layer in micrometers and $h$ is the age of the animal in hours. The slope of this line indicates that the IPL neuropil increases in thickness at an average of $0.02 \mu \mathrm{m}$ per hr until well past metamorphosis. A similar equation for inner nuclear layer thickness is

$$
T_{\text {INL. }}=0.0072 h+24.4
$$

where $T_{\text {INl }}$ is the thickness of the inner nuclear layer in micrometers.

An initial period during which the INL appears to become thinner is followed by a gradual increase in thickness. This initial thinning is not seen in the IPL growth profile.

\section{DISCUSSION}

To express the data as synapses per nucleus, the appropriate number of conventional synapses is divided by the sum of all INL nuclei at that locus whether the nucleus is part of an amacrine, horizontal, bipolar, or Muller cell. The same is done for ribbons and serial synapses. Since rib- bons and conventional synapses define mutually exclusive sets of neurons[ribbons for bipolar neurons, conventional synapses for amacrine neurons (Dowling, 1968) $]$, the estimate given in Figs. 9, 11, 13 of number of synapses per neurons must be less than the true number.

Wong-Riley (1974) has presented evidence that synapses of conventional configuration may also be found within the processes of bipolar neurons. If this is true the interpretation of ribbon-bipolar and conventional-amacrine must be changed. In the many micrographs used in the present study, however, no evidence was seen of ribbons and conventionals in the same process.

A more realistic estimate of the number of synapses per neuron can be calculated if we assume that one-third of the INL nuclei are amacrine cells and one-third are bipolar cells. To obtain this estimate simply multiply the number of synapses per nucleus given on the ordinates of Figs. 9, 11, and 13 by three.

Amacrine cells make both serial and conventional synapses. 'The total number of synapses made per amacrine cell, therefore, is the sum of conventional and serial synapses per cell. Making these calculations, each amacrine cell in the juvenile Xenopus makes, on the average, nearly 10,000 synapses while each bipolar cell makes 1200 ribbon contacts.

These estimates also allow a more realistic rate of synapse production per neuron to be calculated. The mean rate for amacrine cell synapse production is $26.9 \mathrm{syn}$ apses per amacrine cell per $\mathrm{hr}$ between stage 40 and 47 . The rate is 3.6 synapses per cell per hr after stage 47 . Put another way, each amacrine cell at this retinal locus is making, on the average, one new synapse every 2.3 min during early retinal growth, and one every $16.8 \mathrm{~min}$ thereafter.

Bipolar neurons produce ribbon synapses at a rate of 13.7 synapses per $\mathrm{hr}$ during the period of maximal connectivity. This corresponds to one new ribbon syn- 
apse per bipolar cell every $4.4 \mathrm{~min}$, on the average. Once all ribbon synaptic connections are completed, however, the absolute number of synapses per bipolar neuron remains constant even though the neuropil volume is increasing. The average rate of ribbon synapse production at this retinal locus following stage 47 is, therefore, zero.

In order to assess accurately the rates of change of synapses per neuron it is necessary to know if any change occurs by addition or subtraction of neurons. Straznicky and Gaze (1970) have followed the early stages of Xenopus retinal development with autoradiography. They reported no labeling of cells except at the retinal margin after stage 35 . They further report that they find no evidence for extensive degeneration of differentiated retinal neurons.

Hollyfield (1971), however, has reported evidence, again from autoradiography, that labeled nuclei are found in the retinal fundus after $\left[{ }^{3} \mathrm{H}\right]$ thymidine injection at stage 55 . These results indicate that interstitial addition of cells does take place after stage 55 . This is in direct conflict with Gaze's reported findings. The interpretation of per-neuron rates during early synaptic array formation is not altered by this conflict. If, however, additional neurons are added after stage 55 or if neuronal degeneration and death are shown to be important in Xenopus retina, the rate in synapses per neuron would have to be recomputed to reflect the timing and magnitude of these changes.

Interaction of the growth in IPL thickness and the production of synapses results in bipolar and amacrine synaptic arrays which differ in their mode of connectivity. Bipolar neurons maintain a constant number of synapses per neuron at a given locus regardless of the total volume of the neuropil at that locus. Since bipolar neurons are the elements which transmit information into the IPL from the receptors, the constancy of synaptic number per neuron implies that the input to a given IPL locus remains constant once the initial synaptic connections are formed.

Amacrine cells, on the other hand, maintain a constant density of synapses at a given locus. Synapses are formed by these neurons at a rate which is just sufficient to compensate for the growth in volume of the IPL neuropil. Maintenance of a constant density of synapses implies that intersynaptic spacing of conventional synapses is important to the functioning of the synaptic array. To maintain this spacing, the amacrine cells continue the formation of new synapses for as long as the neuropil grows.

In Rana pipiens, serial synapse density increases dramatically at the time of forelimb emergence (Fisher, 1972). This difference in lateral connections within the IPL neuropil is presumably related to a functional difference in the retina between larval and adult Rana. Two observations relevant to this may be noted from the present studies. First, no increase in serial synapses occurs at the time of metamorphosis in Xenopus; and second, even though the density of serial synapses is low in Xenopus, using the estimates explained above, each amacrine cell in the juvenile forms 600 synapses with other amacrine cells, on the average. This number, too, is a low estimate since each synapse of a serial pair must be in the plane of section in order to be counted as serial conventional. A certain fraction of the conventional synapses, therefore, must be one member of a serial synapse the other member of which lies out of the plane of section. From this reasoning, 600 synapses in which the postsynaptic neuron is an amacrine cell must be a low estimate. The true number of synapses must be somewhat higher although the proper correction factor for this sampling error is not available. Even though density of serial synapses is low when compared to conventional density or when compared to density of serial synapses found in adult Rana pipiens (Dowling, 1968; Dubin, 1970; Fisher, 1972), 600 synapses per neuron is a number that 
cannot be ignored when modeling IPL synaptic arrays and their functions.

In a description of ganglion cell development in Xenopus, Chung et al. (1975) describe gradual changes in ganglion cell receptive field properties as the larva matures. No sudden change occurs at metamorphosis. These findings are consistant with the gradual increase in number of amacrine cell synapses reported here. Presumably the increase in synaptic connectivity underlies the reported receptive field changes.

Several investigators have reported quantitative studies of aspects of synaptic array development in other areas of the CNS: rat superior colliculus (Lund and Lund, 1972), rat lateral geniculate (Karlsson, 1966), rat parietal cortex (ArmstrongJames and Johnson, 1970; Aghajanian and Bloom, 1967), rat corpus Striatum (Hattori and McGeer, 1973), cat visual cortex (Cragg, 1969). These studies report synaptic densities in a variety of relative manners such as percentage of adult synapses, synaptic ratios, density of profiles per surface area, or density of profiles per unit volume. Some of the studies purporting to give synaptic density give the numerical density of vesicle-containing processes instead, while others count membrane specializations as synapses. These varying bases for reporting the data make comparison difficult.

In the retinal IPL, Dubin (1970) reports 110 ribbons per $1000 \mu \mathrm{m}^{3}$ and 720 conventional synapses per $1000 \mu \mathrm{m}^{3}$ for the adult Rana pipiens; 135 ribbons per $1000 \mu \mathrm{m}^{3}$ and 1060 conventionals $/ 1000 \mu \mathrm{m}^{3}$ for Rana catesbiana. These compare with 75 ribbons $/ 1000 \mu \mathrm{m}^{3}$ and 625 conventionals $/ 1000$ $\mu \mathrm{m}^{3}$ in Xenopus from the present study. It is tempting to speculate that the quantitative differences found correlate with differences in visually guided behavior found between the species, especially of feeding behavior.

Gaze et al. (1974) report that visually evoked responses can first be recorded from the tectum at stages $43-44$. By stages 45-46 receptive fields are easier to define but are abnormal in their ordering across the tectum. Receptive fields are very large and, in some cases, both temporal and nasal visual fields project to the same tectal locus. The animal from stages $43-46$ is still in the process of adding synapses of amacrine and bipolar origin to the synaptic array of the IPL. At stage 47, when normal ordering of tectal projections is found, the central retina has ceased the production of ribbon synapses and slowed its production of conventional synapses to a rate which maintains a constant density. It would appear, therefore, that central terminations of ganglion cells within the tectum are sorted out at the same time as the amacrine and bipolar synapses are formed within the retina.

The light microscopic appearance of the receptors and outer plexiform layer in this study are essentially the same as described by Saxen (1954).

Several features of the normal development of synaptic arrays have been characterized in this study: the number of ribbon and conventional synapses per neuron have been calculated; the rate of formation of synapses per neuron per unit time has been derived; the density of synapses per unit volume as a function of time has been determined; the time of the major change in rate of synapse production has been identified; and a fundamental difference between the modes of continuing connectivity of conventional and ribbon synapses has been observed. Each of these features might be used in experimental studies to gain insight into parameters which affect synaptic connectivity. For example, does introduction of a specific hormone change the rate of production of synapses per neuron? Would it change the location of the breakpoint in the growth profiles? Are total numbers of synapses per neuron changed by environmental factors? There is some indication that synaptic densities are different in light-reared and dark- 
reared Xenopus (Tucker, 1975).

These questions point out the usefulness of a quantitatively defined normal system for the continuing study of the development of the nervous system. Changes in the nervous system which affect its final configuration may well be quantitative rather than qualitative. Experimental manipulation of factors which may affect the development of synaptic arrays can be assayed on a quantitative basis using the normal data presented here.

I thank Linda Jamison and Sharon Burke for their excellent technical assistance, and S. S. Easter, Jr., for his encouragement and invaluable critical advice. This research was supported, in part, by NSF Grant Number GB41553, and University of Michigan Rackham Grant Number 360129.

\section{REFERENCES}

Aghajanian, G. K., and Bloom, F. E. (1967). The formation of synaptic junctions in developing rat brain: A quantitative electron microscopic study. Brain Res. 6, 716-721.

Armstrong-JAmes, M., and Johnson, R. (1970). Quantitative studies of postnatal changes in synapses in rat superficial motor cerebral cortex. $Z$. Zellforsch. 110, 559-568.

Chung, S. H., Stirling, R. V., and Gaze, R. M. (1975). The structural and functional development of the retina in larval Xenupus. J. Embryol. Exp. Morph. 33, 915-940.

CragG, B. G. (1974). Plasticity of synapses. Brit. Med. Bull. 30, 141-144.

Dowling, J. E. (1968). Synaptic organization of the frog retina: An electron microscopic analysis comparing the retinas of frogs and primates. Proc. Roy. Soc. 170, 205-228.

Dubin, M. W. (1970). The inner plexiform layer of the vertebrate retina: A quantitative and comparative electron microscopic analysis. J. Comp. Neurol. 140, 479-506.

EbBesson, S. O. E., and TANG, D. (1965). A method for estimating the number of cells in histological sections. J. R. Microse. Soc. 84, 449-469.

Fisher, L. J. (1972). Changes during maturation and metamorphosis in the synaptic organization of the tadpole retina inner plexiform layer. Nature (London) 235, 391-393.

Gaze, R. M., Keating, M. J., and Chung, S. H. (1974). The evaluation of the retinotectal map during development in Xenopus. Proc. Roy. Soc. Lond. Ser. $B$ 185, 301-330.

Hattori, T., and McGeer, P. L. (1973). Synaptogenesis in the corpus striatum of infant rat. Exp.
Neurol. 38, 70-79.

Hollyfield, J. G. (1971). Differential growth of the neural retina in Xenopus laevis larvae. Devel. Biol. 24, 264-286.

KARLSSON, U. (1967). Observations on the postnatal development of neuronal structures in the lateral geniculate nucleus of the rat by electron microscopy. J. Ultrastr. Res. 17, 158-175.

LuND, R. D., and LuND, J. S. (1972). Development of synaptic patterns in the superior colliculus of the rat. Brain Res. $42,1-20$.

Nace, G. W., Culley, D. D., Emmons, M. B., Gibbs, E. L., Hutchison, V. H., and McKinnell, R. G. (1974). "Amphibians: Guidelines for the Breeding, Care and Management of Laboratory Animals." National Academy of Sciences, Washington, D.C.

Nieuwkoop, P. D., and FABer, J. (1967). "Normal Table of Xenopus laevis (Daudin)." North Holland Publishing Co., Amsterdam.

Nilsson, S. E. G., and Crescitelli, F. (1969). Changes in ultrastructure and electroretinogram of bullfrog retina during development. J. Ultrastr. Res. 27, 45-62.

Nilsson, S. E. G., and Crescitelli, F. (1970). A correlation of ultrastructure and function in the developing retina of the frog tadpole. J. Ultrastr. Res. 30, 87-102.

Olney, J. W. (1968). An electron microscopic study of synapse formation, receptor outer segment development, and other aspects of developing mouse retina. Invest. Ophthalmol. 7, 250-268.

Peachey, L. D. (1958). Thin sections. I. A study of section thickness and physical distortion produced during microtomy. J. Biophys. Biochem. Cytol. 4, 233.

SAXEN, L. (1954). The development of the visual cells. Ann. Acad. Sci. Fennicae 23.

Sosula, L., and Glow, P. H. (1970). A quantitative ultrastructural study of the inner plexiform layer of the rat retina. J. Comp. Neur. 140, 439-478.

Straznicky, K., and Gaze, R. M. (1971). The growth of the retina in xenopus laevis: An autoradiographic study. J.Embryol. Exp. Morph. 26, 6779.

Tucker, G. S. (1975). Responses of Inner plexiform synapses to dark rearing in the retina of the toad, Xenopus laevis. Neuroscience (Abstracts) 1, 98.

Weidman, T. A. M., and Kuwabara, T. (1968). Postnatal development of the rat retina. Arch. Ophthal. 79, 470-484.

West, R. W. (1972). Thick sections of epon. Stain Technol. 47, 201.

WONG-RILEY, M. T. T. (1974). Synaptic organization of the inner Plexiform layer in the retina of the tiger salamander, J. Neurocytol. 3, 1-33.

YAZULLA, S. (1974). Intraretinal differentiation in the synaptic organization of the inner plexiform layer of the pigeon retina. J. Comp. Neur. 153, 309- 324 